\title{
PREPRINT
}

\section{Arabic digit processing in adults with mathematical learning disability}

Samuel Lepoittevin ${ }^{1}$, Gaétane Keymolen ${ }^{1}$, Michael Andres ${ }^{1,2} \&$ Alice De Visscher ${ }^{3}$

${ }^{1}$ Psychological Sciences Research Institute, Université catholique de Louvain, Belgium.

${ }^{2}$ Institute of Neuroscience, Université catholique de Louvain, Belgium.

${ }^{3}$ Research Center in the Psychology of Cognition, Language, and Emotion (PsyCLE), Université AixMarseille, France.

The final version of this work has been published and can be found at: https://journals.sagepub.com/doi/10.1177/17470218221128498

\section{Please refer to the final published version}




\section{Corresponding author:}

Samuel Lepoittevin

Institut de Recherche en Sciences Psychologiques

Université catholique de Louvain

Place Cardinal Mercier, 10

1348 Louvain-la-Neuve

Belgium

Email : samuel.lepoittevin@uclouvain.be

\section{Credit authorship contribution statement}

SL: Conceptualization, Methodology, Investigation, Formal analysis, Data curation, Writing - original draft, Writing - review \& editing, Visualization. GK: Conceptualization, Methodology, Investigation. MA: Conceptualization, Writing - original draft, Writing - review \& editing. ADV: Conceptualization, Methodology, Formal Analysis, Writing - original draft, Writing - review \& editing, Visualization, Supervision, Project administration, Funding acquisition. 


\begin{abstract}
Processing Arabic digits is a core difficulty for children suffering from mathematical learning disability (MLD). Dominant accounts assume a semantic impairment affecting either the magnitude representation per se or its access from numerical symbols. But recent data have raised the alternative hypothesis of a selective deficit of digit visual recognition (i.e., recognizing a symbol as one of the digits, no matter its identity or numerical meaning). This study aims at testing whether the difficulty to process Arabic digits remains prevalent in adults with MLD and whether it is effectively associated with a digit visual recognition deficit. To do so, we compared 19 adults with MLD to 19 matched controls in an Arabic digit comparison task that required to identify the largest of two digits, and in an Arabic digit lexical decision task that required to decide whether a visual stimulus is a digit or not. The results showed that MLD participants took more time than control participants to perform the comparison task. In contrast, their performance in the digit lexical decision task was within the range of controls. Overall, these findings indicate that adults with MLD continue to experience difficulties to process the magnitude of Arabic digits efficiently, and that this cannot be explained by a visual recognition deficit for Arabic digits. We conclude that their difficulties are best explained by an impaired representation of number magnitude or by an impaired access to this representation.
\end{abstract}

Keywords: Dyscalculia, Symbolic number comparison, Lexical decision, Visual recognition 
Mathematical learning disability (MLD) is a specific, severe and persistent disorder of numerical development and mathematical learning (American Psychiatric Association, 2013), which would affect from $3 \%$ to $6 \%$ of the population (e.g., Shalev et al., 2000), depending on the inclusion threshold used, the testing instruments, and the numerical domains covered by the testing (e.g., arithmetical skills, mathematical conceptual knowledge). Most researchers agree on the heterogeneity of the disorder, which may affect different skills (Geary, 2004), such as transcoding from a symbolic code to another (Sullivan, 1996; Temple, 1989), learning and retrieving arithmetic facts (De Visscher \& Noël, 2013; Geary et al., 2000), reciting the number sequence (Landerl et al., 2004), or using mature calculation strategies (Geary et al., 2000).

A common observation among studies is that children with MLD seem to experience difficulties whenever they have to process numerical symbols (Schwenk et al., 2017), even in basic tasks such as magnitude comparison (see De Smedt et al., 2013, for a review). It is unclear whether these difficulties persist after schooling but a few observations suggest they still influence the comparison skills of adults with MLD (Wilson et al., 2015). This has prompted the hypothesis that inefficient processing of numerical symbols is a core feature of MLD. However, the nature of the underlying deficit is debated. According to the dominant account, this would be the expression of a general impairment affecting the approximate numerical system (ANS; Wilson \& Dehaene, 2007), which is defined as an innate and phylogenetically inherited magnitude processing system whose precision is crucial for mathematical development because this system would provide a common ground for symbolic and non-symbolic number skills (Mazzocco et al., 2011a; Booth \& Siegler, 2008). Supporting evidence comes from the finding that the precision of the ANS, as indexed by the discrimination ratio between two numerosities (e.g., dot collections), is lower in children with MLD than in age-matched control children (Mazzocco et al., 2011b; Piazza et al., 2010). Discrepant results come from studies reporting serious difficulties in Arabic digit comparison in a context of normal performance in dot collection comparison. The finding of disproportionate difficulties with symbolic vs. non-symbolic magnitude comparison does not match the role assigned to the ANS in MLD (De Smedt \& Gilmore, 2011; Iuculano et al., 2008; Landerl \& Kölle, 2009; Rousselle \& Noël, 2007). Alternative accounts imply a selective deficit in the processing 
of symbolic numbers. The first account builds on models of numerical cognition where symbolic number representations (i.e., Arabic digits, number words) converge to a core representation of magnitude (Dehaene, 1992; McCloskey et al., 1985). Rather than the representation of numerical magnitude per $s e$, it would be the access to this representation from symbols that is impaired, hence disrupting the matching of Arabic digits with their respective magnitude (Rousselle \& Noël, 2007). The second account considers that symbolic and non-symbolic comparisons rely on distinct representations that could be selectively impaired in MLD. Some behavioural (e.g., Marinova et al., 2021) and neurophysiological data (e.g., Roggeman et al., 2007) support the existence of distinct representations for numerosity (i.e., dots) and numerals (i.e., digits). Under this account, the acquisition of the symbolic code would lead to the elaboration of an exact representation that links the position of numbers in the verbal sequence to the cardinality they represent (Carey, 2001, 2009; Reynvoet \& Sasanguie, 2016). Throughout development, this exact representation would map onto the approximate representation inherited from evolution to estimate numerosity, leading to its refining (Piazza et al., 2013). The main difference with the ANS theory is that the interaction between non-symbolic and symbolic numbers goes in the opposite direction. The refinement hypothesis is supported by empirical data showing that MLD is characterized by early difficulties in the processing of Arabic digits (i.e., before the age of ten), followed later on by difficulties signaling a departure of the non-symbolic number system from the normal developmental trajectory (Noël \& Rousselle; 2011).

In sharp contrast with those semantic accounts lies the possibility that children with MLD have difficulties to process Arabic digits efficiently because their visual recognition of digits, (defined as the ability to match a percept as one of the Arabic digits, no matter its identity or meaning), is particularly challenging and takes more time. The best way to characterize this pre-semantic account is to refer to the allographic representation in reading models (Schubert \& McCloskey, 2013; McCloskey \& Schubert, 2014). This representation finds a counterpart in numerical cognition models under the visual Arabic code that has been distinguished from the verbal and analogical codes (Dehaene, 1992; Dehaene \& Cohen, 1995). This processing stage is typically assessed through digit lexical decision tasks (McCloskey \& Schubert, 2014), which consist in deciding whether a symbol (digit or pseudo-digit) is a 
digit or not, irrespective of its identity (and thus without requiring any semantical or transcoding processing). A recent study showed that 8-9-year-old children with MLD committed more errors in this simple digit lexical decision task than age-matched control children, suggesting an impairment of digit recognition ability (Lafay et al., 2018). However, firm conclusions are prevented by the heterogeneity of the groups and by the methodology used to assess digit recognition at a pre-semantic level. Indeed, the group difference disappeared when standard measures of linguistic and non-verbal reasoning skills were introduced as covariates. Moreover, their digit lexical decision task involved other characters as distractors (letters and arithmetical signs). The performance thus reflects the ability to discriminate digits from other characters, which corresponds to a subsequent processing stage that deals with the character category (McCloskey \& Schubert, 2014). Therefore, it remains unclear whether the difficulties that characterize the processing of Arabic digits in MLD can be assigned to a visual recognition deficit. Finally, we have underlined the need to complement research on MLD with data from adults in order to get a better idea of the pervasive nature of this disorder.

The present study aims to fill this gap through an investigation of Arabic digit processing in adults with MLD. The study includes magnitude comparison and digit lexical decision tasks to distinguish between semantic and pre-semantic stages of processing, respectively. The Arabic digit lexical decision task required participants to decide whether a sign was a digit or not, and pseudo-digits - rather than other symbols - were used as distractors to avoid the involvement of further categorical processing. For the sake of comparison, we also tested participants in a letter lexical decision task where they had to decide whether the sign was a letter or not. We predict that (1) adults with MLD should experience difficulties in the Arabic digit comparison task given that the symptoms of MLD tend to persist over time (Wilson et al., 2015), and that (2) these difficulties should be accompanied by a poor performance in the digit lexical decision task if they originated from a pre-semantic deficit (Lafay et al., 2018). 


\section{Method}

\section{Participants}

Participants were native French-speaking adults, who gave their written consent. They all met the two following inclusion criteria: (1) being aged between 18 and 40 years; (2) being exempt from history of neurological or psychiatric disease, and performing in the standard range (i.e., standard note within [4-16]) in the following two subtests of the French edition of the WAIS-IV battery (Wechsler, 2011): "Similitudes" (verbal reasoning skills) and "Symbole" (processing speed). Participants with MLD were included if they reported long-lasting mathematical difficulties, which appeared during the first two years of primary school and had persisted since then, and if they performed below the normative mean (Z-score $<-1.5)$ in at least one of the following tests: the Arithmetic Battery calculation tests (Shalev et al., 2001, modified by Rubinsten \& Henik, 2005), and The TempoTest Automatiseren (TTA; de Vos, 2010). Thirty-one adults volunteered, but 11 did not meet the selection criteria (4 because of their performance in the WAIS-IV subtests and 7 reported math difficulties that did not meet the severity criterion in the arithmetic tests). We thus included 20 MLD participants in the study. One of them had to be excluded from the subsequent analyses because his median reaction time in one of the computerized control tasks was identified as an outlier observation that compromised the normal distribution of the data. The control group (CG) included adults matched with the MLD participants in terms of age, gender, lateralization, and educational level (number of completed school years). They were selected if they performed in the standard range in the subtests of the WAIS-IV battery and had no history of mathematical learning difficulty, as further evidenced by Z-scores $\geq-1$ in the two arithmetic tests. Nine volunteers did not meet the inclusion criteria regarding arithmetic performance and were replaced by other participants. The control participant matched with the outlier participant from the MLD group was excluded from subsequent analysis as well, to obtain two groups of 19 participants with comparable socio-demographic profiles and equal non-verbal reasoning skills (see Table 1). 
Table 1: Description of MLD and CG groups.

\begin{tabular}{|c|c|c|c|c|}
\hline & MLD & CG & Tests & $p$ \\
\hline \multicolumn{5}{|l|}{ Socio-demographic variables } \\
\hline Sex & $19+$ & 19 ㅇ & & \\
\hline Manual dominance & $16 \mathrm{R} ; 3 \mathrm{~L}$ & $16 \mathrm{R} ; 3 \mathrm{~L}$ & & \\
\hline Age $(y-o)$ & $M=23.18, S D=2.32$ & $M=23.09, S D=2.26$ & $t(36)=.13$ & $p=.901$ \\
\hline Number of school years & $M d n=15$ & $M d n=15$ & $U=162$ & $p=.603$ \\
\hline \multicolumn{5}{|l|}{ WAIS IV Measures } \\
\hline WAIS "Similitudes" & $M d n=12$ & $M d n=14$ & $U=130$ & $p=.146$ \\
\hline WAIS “Symboles” & $M d n=10$ & $M d n=12$ & $U=86$ & $p=.005$ \\
\hline \multicolumn{5}{|l|}{ Arithmetic Measures } \\
\hline Arithmetic battery accuracy (z) & $M d n=-1.50$ & $M d n=0.52$ & $U=41$ & $p<.001$ \\
\hline Arithmetic battery speed (z) & $M=-2.09, S D=1.65$ & $M=.29, S D=.65$ & $t(23.49)=-5.85$ & $p<.001$ \\
\hline TTA addition/subtraction fluency (z) & $M d n=-1.19$ & $M d n=1.19$ & $U=9$ & $p<.001$ \\
\hline TTA total fluency (z) & $M=-2.17, S D=1.20$ & $M=.69, S D=.68$ & $t(36)=-9.05$ & $p<.001$ \\
\hline
\end{tabular}

\section{Task and Procedure}

The study was approved by the ethical committee of the Psychological Sciences Research Institute of UCLouvain (Belgium). Experimental tasks were programmed using PsychoPy3 (Version 3.1.5; Pierce, 2019) and run on a laptop computer with a 15.6-inch screen (1920 x 1080 resolution; 60 $\mathrm{Hz}$ refresh rate). The screen was positioned $60 \mathrm{~cm}$ from the participants eyes, so that the relative size of the displayed stimuli allowed their apprehension by the fovea $\left(<1^{\circ}\right.$ from central position). Stimuli were images with a grey background, sharing the same resolution and original size. Before each task, written instructions were displayed on screen. Participants were asked to answer by pressing the left or right response key corresponding to the key "S" or "L" on the keyboard. The instructions emphasized both speed and accuracy. In each trial, the stimulus was preceded by a 500-ms fixation cross and remained on screen until the participant's response. The inter-trial-intervals were set to 500ms. After collecting socio-demographic data, screening the participants for their arithmetic, processing speed and non-verbal reasoning skills, we administered the experimental tasks in the following order, for a total duration of 120 minutes, including short breaks in-between the tasks.

Arabic digit comparison task. The first experimental task was a computerized Arabic digit comparison task (COMPARISON). Stimuli consisted in the 72 possible pairs of digits, presented twice, for a total of 144 trials per subject. They remained on screen until response. Participants had to respond, as 
quickly as possible, by pressing the response key on the side of the digit with the largest numerical magnitude (see Figure 1A). The side of the correct response was counterbalanced across trials. The trial order was pseudo-randomized, so that participants did not respond more than 3 times in a row with the same key, and the same stimulus did not appear 2 times in a row. The task started with a practice session of 8 trials.

Lexical decision task on Arabic digits. In the computerized digit lexical decision task (DIGITDECISION), participants had to decide whether a given stimulus was a digit or not. The digits (Calibri) ranged from 1 to 9 , excluding 8 (see Figure 1B). Three sets of pseudo-digits were created by modifying the spatial arrangement of the features constitutive of each digit, while avoiding discontinuity or overlap, to end up with the same physical characteristics as the original digits (i.e., number of salient features, density). To make the task more sensitive to interindividual differences, we increased the task difficulty by adding a noisy background in half of the trials $\left(0.5^{\circ}\right.$ noise filter available from the Psychopy component library). Stimuli remained on screen until response. Participants had to respond as fast as possible. Half of the MLD participants - and of the matched controls - responded to the digits with the left key and to the pseudo-digits with the right key, whereas the other half of the participants received the opposite response key assignment. The task counted 48 trials with a transparent background and 48 with a noisy background so that each digit was repeated three times and each pseudo-digit once in each condition. The trial order was pseudo-randomized, so that participants did not respond more than three times in a row with the same key, the same background did not appear more than two times in a row, and stimuli originated from the same digit did not appear two times in a row. The task started with a practice session of 8 trials.

Lexical decision task on letters. As a control condition, we designed another lexical decision task that required participants to decide whether a given stimulus was a letter or not (LETTER-DECISION). The task was identical in all aspects to the DIGIT-DECISION task, except that the stimuli (lower case, Calibri) consisted in letters (a, e, f, h, k, r, s, t) and pseudo-letters created using the same method as for pseudo-digits. 
Choice reaction time tasks. The COMPARISON and DIGIT-DECISION tasks were each supplemented with a matched choice reaction time task (respectively CRT-COMP and CRT-DEC) designed to account for the inter-individual variability in response speed resulting from domain-general processes such as perception, attention or motor preparation (see Figure 1). In the CRT-COMP task, the stimuli consisted in pairs of red and blue rectangles, displayed side by side, as the digit pairs in the COMPARISON task. This task required participants to press the response key on the side of the red rectangle. In the CRT-DEC task, a red or blue rectangle was displayed alone at the center of the screen as the single characters presented in the LEXICAL DECISION tasks. Participants were asked to decide whether the rectangle was red or not by pressing the correct response key. The two CRT tasks started with 4 practice trials followed by 30 test trials.

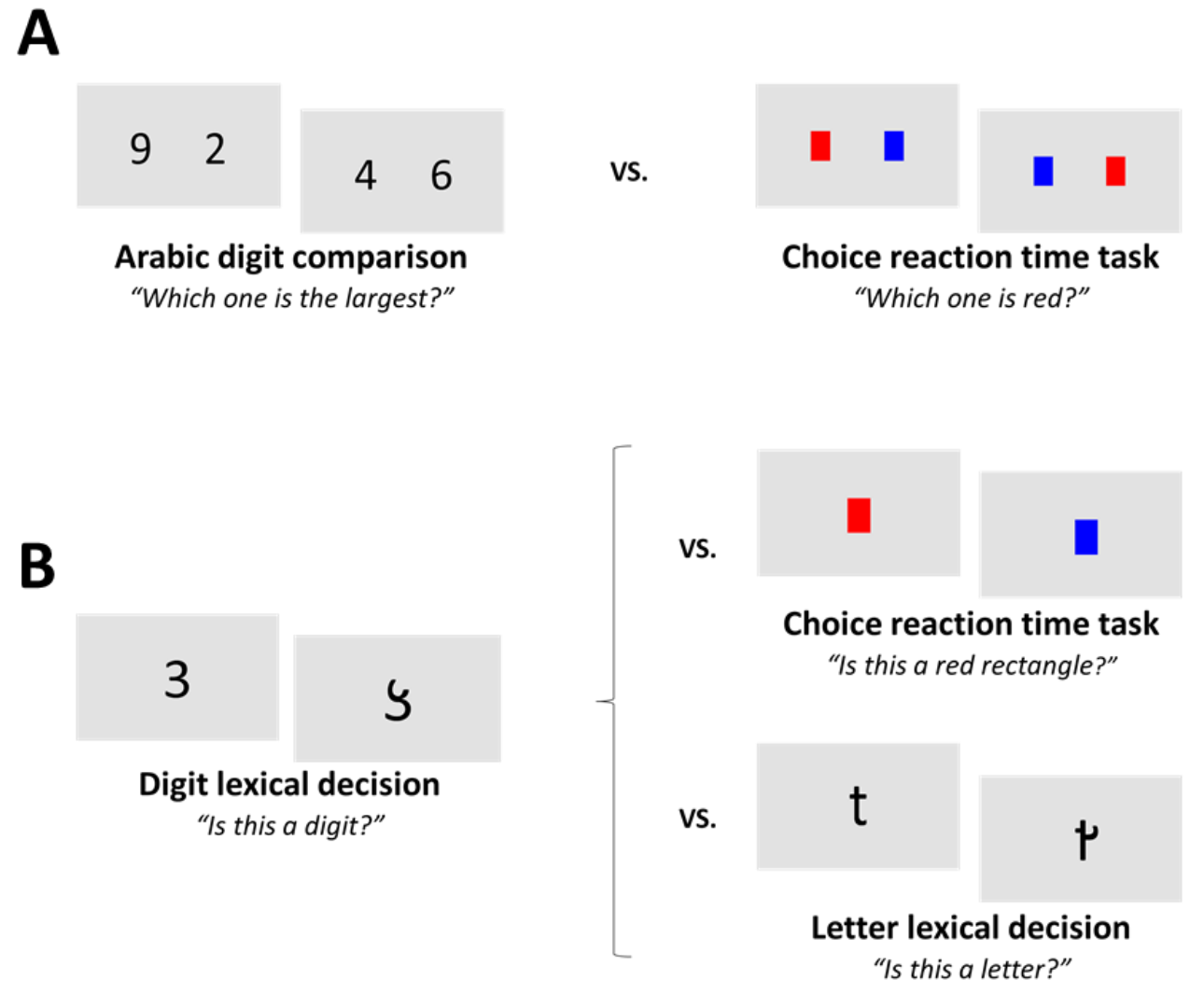

Figure 1. Examples of stimuli in the Arabic digit comparison task (A) and in the digit lexical decision task (B), and in their respective control conditions (A: CRTCOMPARISON; B: CRT-DECISION and LETTER-DECISION) 


\section{Data Analysis}

The median RT on correct trials was calculated for each participant and each task. Errors were not analyzed because the mean accuracy ranged from 96 to $98 \%$ across tasks (see Table 2). The RT data were log-transformed to obtain a normal distribution in each task and condition. The normal distribution was tested using Shapiro-Wilk (all $p \mathrm{~s}>.05$ after transformation) and, when relevant, the homogeneity of variances was verified and the residuals were analyzed to exclude any other abnormality in the distribution of the data. The untransformed RT data are reported in Table 2 and can be visualized on Figures 2, 4 and 5. In order to investigate group differences, we conducted a $2 \times 2$ ANOVA on the $\log _{10}(\mathrm{RT})$ with Task (COMPARISON or DECISION vs. CRT-COMP or CRT-DEC) as within-subjects factor and Group (MLD vs. CG) as between-subjects factor. Bonferroni correction was applied in post-hoc analyses. These group analyses were complemented with individual data analyses. For Arabic digit comparison, we regressed the RTs of the CG participants in the COMPARISON task as a function of their RTs in the matched control task (CRT-COMP). We then computed a 95\% confidence interval around the regression line and identified the MLD participants whose RTs fell outside this interval on the XY graph (Figure 3). For Arabic digit recognition, we computed the difference in reaction time (dRTs) between the DIGIT-DECISION and CRT-DECISION tasks. We then ranged the dRTs of all participants from the lowest to the highest and examined whether the participants with MLD - and more specifically those who had difficulty in Arabic digit comparison - were in the range of control participants or not (Figure 5). Finally, to further explore the selectivity of difficulties in visual recognition, we directly compared digits and letters considering the influence of the background noise. The $\log _{10}(\mathrm{RT})$ of both lexical decision tasks were entered in a $2 \times 2 \times 2$ ANOVA with lexical decision Task (digits vs. letters) and Background (transparent vs. noisy) as within-subjects factors and Group (MLD vs. CG) as betweensubjects factor. 
Table 2: Mean and standard deviation of the individual accuracy rates (ACC) and correct reaction times (RT) as a function of group and task.

\begin{tabular}{|c|c|c|c|c|c|c|c|c|c|c|c|}
\hline & \multirow{2}{*}{$\mathbf{N}$} & \multicolumn{2}{|c|}{ CRT-COMP } & \multicolumn{2}{|c|}{ CRT-DEC } & \multicolumn{2}{|c|}{ COMPARISON } & \multicolumn{2}{|c|}{ DIGIT-DECISION } & \multicolumn{2}{|c|}{ LETTER-DECISION } \\
\hline & & $A C C$ & $R T$ & $A C C$ & $R T$ & $A C C$ & $R T$ & $A C C$ & $R T$ & $A C C$ & $R T$ \\
\hline MLD & 19 & $\begin{array}{l}98.6 \\
(2.8)\end{array}$ & $\begin{array}{l}380.7 \\
(36.5)\end{array}$ & $\begin{array}{l}97.0 \\
(2.9)\end{array}$ & $\begin{array}{l}434.7 \\
(39.7)\end{array}$ & $\begin{array}{l}96.5 \\
(3.4)\end{array}$ & $\begin{array}{l}573.5 \\
(90.9)\end{array}$ & $\begin{array}{l}96.5 \\
(2.9)\end{array}$ & $\begin{array}{l}605.3 \\
(52.5)\end{array}$ & $\begin{array}{l}96.7 \\
(2.6)\end{array}$ & $\begin{array}{l}610.2 \\
(56.3)\end{array}$ \\
\hline CG & 19 & $\begin{array}{l}98.6 \\
(2.0)\end{array}$ & $\begin{array}{l}352.0 \\
(27.0)\end{array}$ & $\begin{array}{l}96.5 \\
(4.2)\end{array}$ & $\begin{array}{l}388.5 \\
(34.7)\end{array}$ & $\begin{array}{l}96.5 \\
(2.6)\end{array}$ & $\begin{array}{l}438.8 \\
(52.7)\end{array}$ & $\begin{array}{l}96.9 \\
(2.2)\end{array}$ & $\begin{array}{l}549.0 \\
(75.6)\end{array}$ & $\begin{array}{l}97.6 \\
(1.9)\end{array}$ & $\begin{array}{l}541.4 \\
(54.0)\end{array}$ \\
\hline
\end{tabular}

Note. ACC are expressed in \%; RT in $\mathrm{ms}$.

\section{Results}

\section{Comparison of Arabic digits}

The ANOVA revealed a main effect of Task $\left(F(1,36)=235.15, p<.001, \eta^{2}{ }_{\mathrm{p}}=.867\right)$, with faster RTs in the CRT-COMP task $(M=366 \mathrm{~ms}, S D=35 \mathrm{~ms})$ than in the COMPARISON task $(M=506 \mathrm{~ms}, S D=$ $100 \mathrm{~ms})$, and a main effect of Group $\left(F(1,36)=30.26, p<.001, \eta^{2} \mathrm{p}=.457\right)$, with faster RTs in CG $(M=$ $395 \mathrm{~ms}, S D=60 \mathrm{~ms})$ than in MLD participants $(M=477 \mathrm{~ms}, \mathrm{SD}=119 \mathrm{~ms})$. The analysis also revealed a significant Group by Task interaction $\left(F(1,36)=21.29, p<.001, \eta^{2} \mathrm{p}=.372\right)$. Post-hoc comparisons showed that CG answered faster than MLD in either task (CRT-COMP: $t(36)=2.78, p=.027$; COMPARISON: $t(36)=5.94, \mathrm{p}<.001)$. However, the difference in RT between the two tasks was larger in the MLD $(M=193 \mathrm{~ms}, S D=87 \mathrm{~ms})$ than in the CG $(M=87 \mathrm{~ms}, S D=39 \mathrm{~ms} ; t(36)=5.69, p<.001)$, pointing to specific difficulties in Arabic digit magnitude comparison (see Figure 2). 


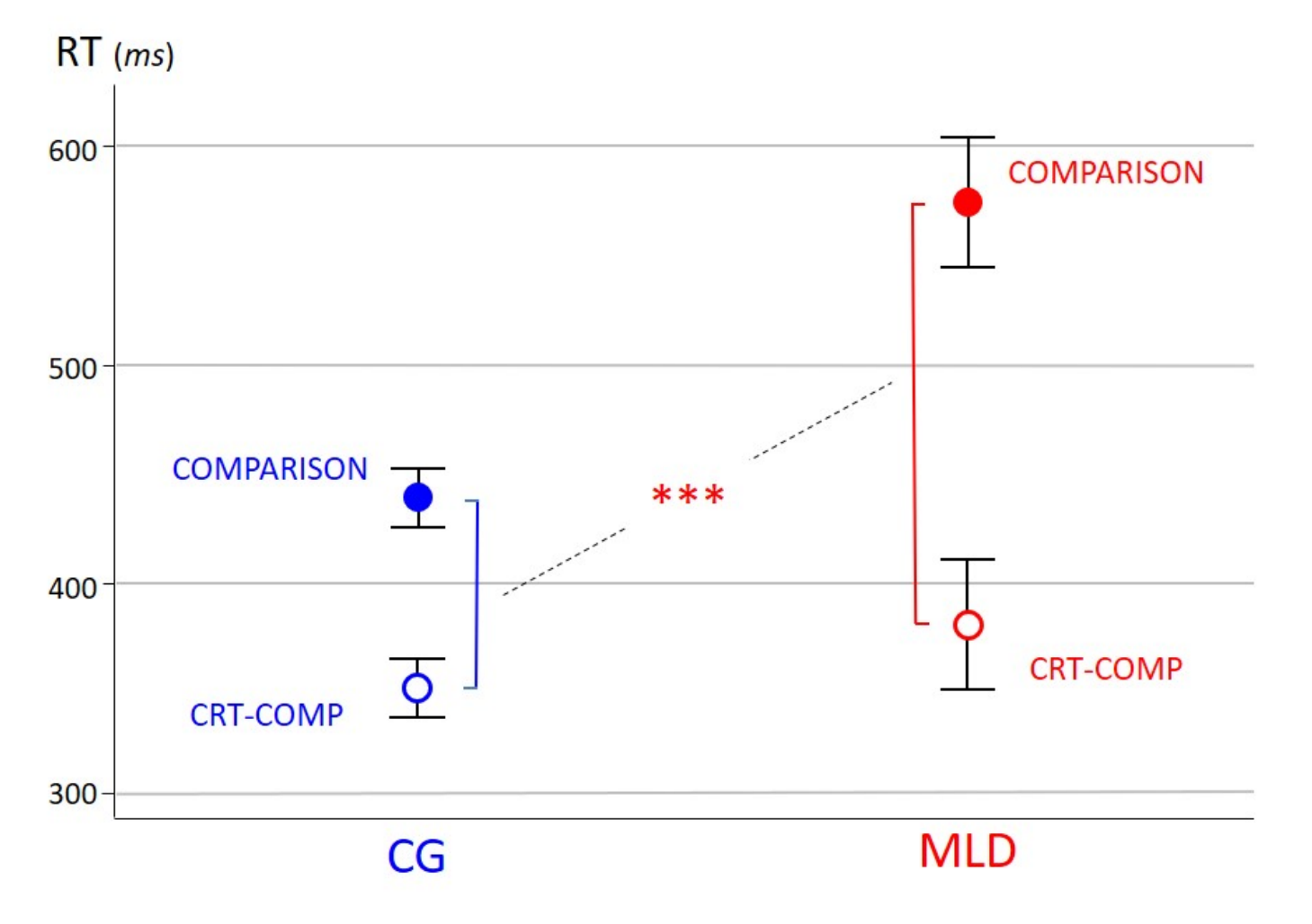

Figure 2. Mean reaction time (RT) in the Arabic digit comparison task (COMPARISON), relative to the related choice reaction time task (CRT-COMP), in the control group (CG) and in the group of participants with mathematical learning disability (MLD). Participants with MLD show a specific increase of their RT in the Arabic digit comparison task compared to matched controls. *** $\mathrm{p} \leq .001$

The analysis of individual data indicated that 7 out of 19 MLD participants are slower in the COMPARISON task than what could be expected from their RT in the matched choice reaction time task. Figure 3 clearly shows that the RTs of these MLD participants are out of the 95\% confidence interval along the regression line describing the performance of the CG participants (COMPARISON $\log _{10}(\mathrm{RT})$ $=-0.2+11.1 *$ CRT-COMP $\left.\log _{10}(\mathrm{RT}), t=4.093, p<.001\right)$. 


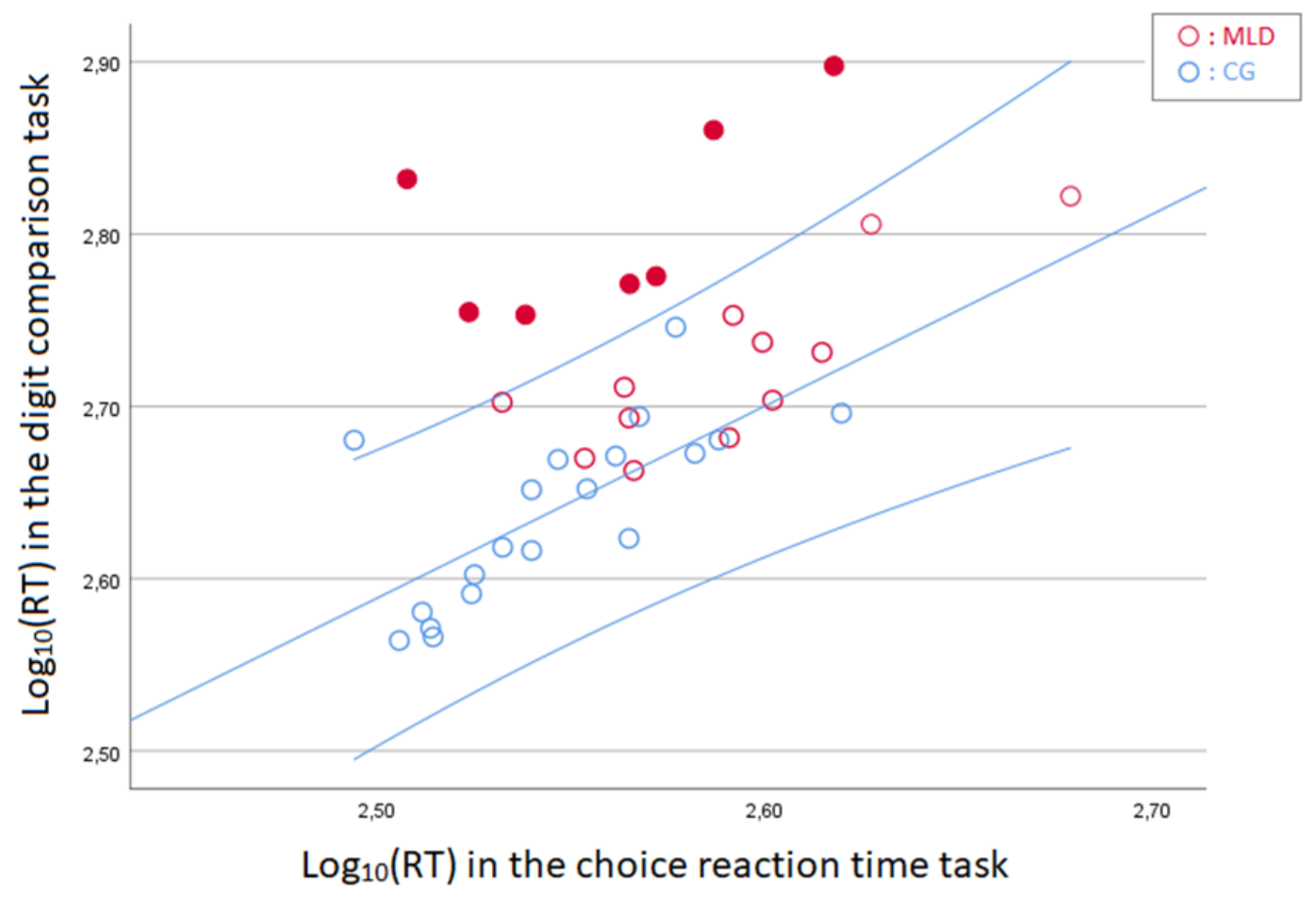

Figure 3. Distribution of reaction times (RT) in the Arabic digit comparison task, with respect to the matched choice reaction time task, for the participants with mathematical learning disability (MLD) and for those of the control group (CG). Seven out of 19 participants with MLD (filled red dots) are out of the range of CG (blue lines).

\section{Visual recognition of Arabic digits}

The first ANOVA, with Task (DIGIT-DECISION vs. CRT-DEC) as within-subjects factor and Group (MLD vs. CG) as between-subjects factor, revealed a main effect of Task $(F(1,36)=277.24, p<.001$, $\left.\eta^{2}{ }_{\mathrm{p}}=.885\right)$, with faster RTs in the CRT-DEC task $(M=412 \mathrm{~ms}, S D=44 \mathrm{~ms})$ than in the DIGIT-DECISION $\operatorname{task}(M=577 \mathrm{~ms}, S D=70 \mathrm{~ms})$. We also found a main effect of Group $\left(F(1,36)=16.20, p<.001, \eta^{2}{ }_{\mathrm{p}}=\right.$ $.310)$, indicating that $C G$ participants $(M=469 \mathrm{~ms}, S D=100 \mathrm{~ms})$ responded faster than MLD participants $(M=577 \mathrm{~ms}, S D=119 \mathrm{~ms})$. However, there was no significant interaction between Group and Task $(F(1,36)=.05, p=.825)$, meaning that the slower performance of MLD was not specific to the digit lexical decision task (see Figure 4). 


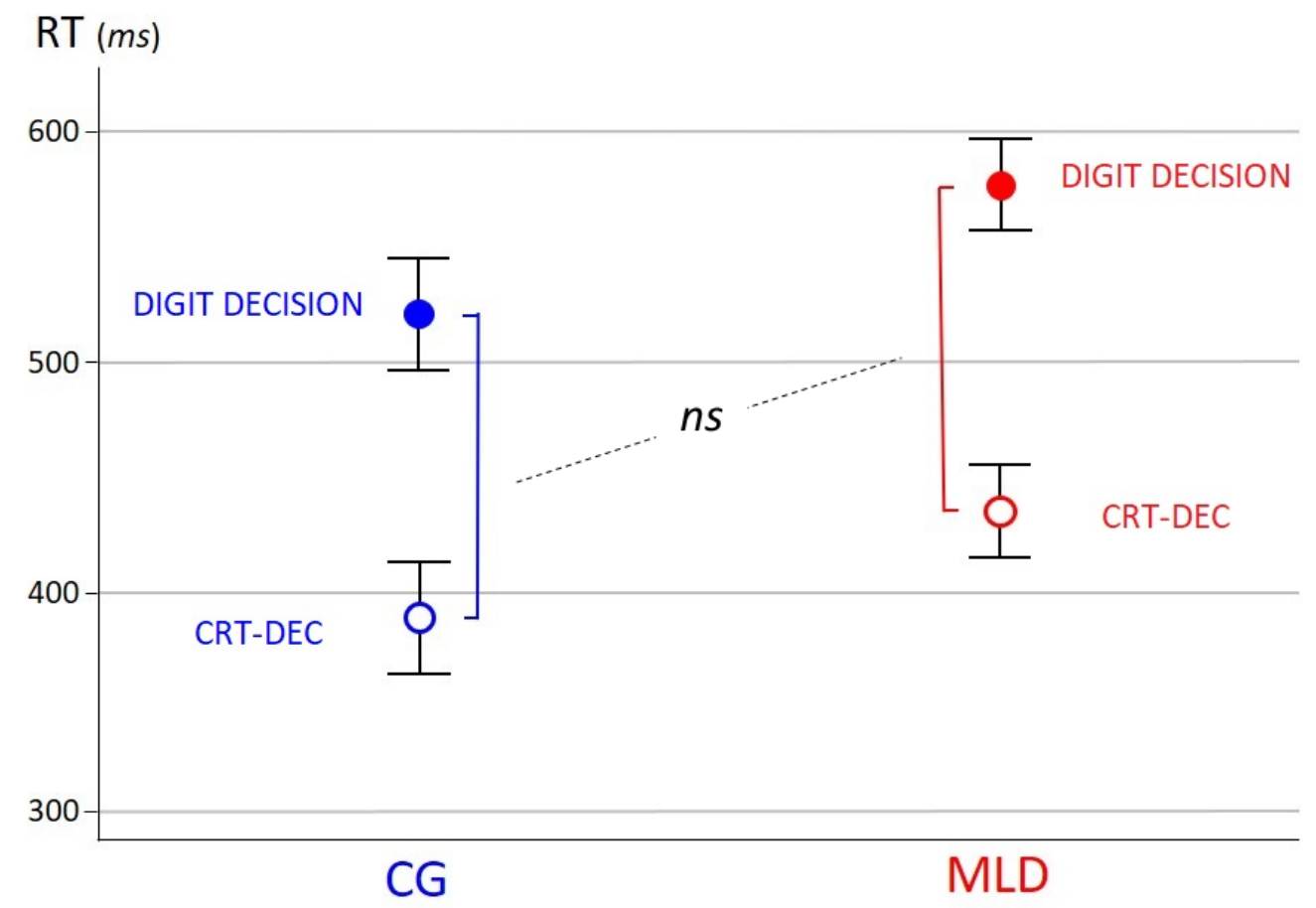

Figure 4. Mean reaction time (RT) in the digit lexical decision task (DIGIT-DECISION), relative to the choice reaction time task (CRT-DEC), in the control group (CG) and in the group of participants with mathematical learning disability (MLD). Participants with MLD show no specific increase of their RT in the digit lexical decision task compared to matched controls.

In order to exclude a deficit in digit visual recognition specifically in the MLD participants that were the most impaired in the COMPARISON task, we analysed the individual difference in reaction time (dRTs) between the DIGIT-DECISION and CRT-DECISION tasks. Figure 5 shows that the dRTs of the participants with MLD, including the MLDs who exhibited a specific slowdown in the COMPARISON task, are within the range of the dRTs of the control participants: several CG participants actually responded slower than these MLDs in the DIGIT-DECISION task (after controlling for response speed differences using a matched choice reaction time task as a baseline). Thus, the dissociation between impaired digit comparison and preserved digit recognition also holds at the individual level. 


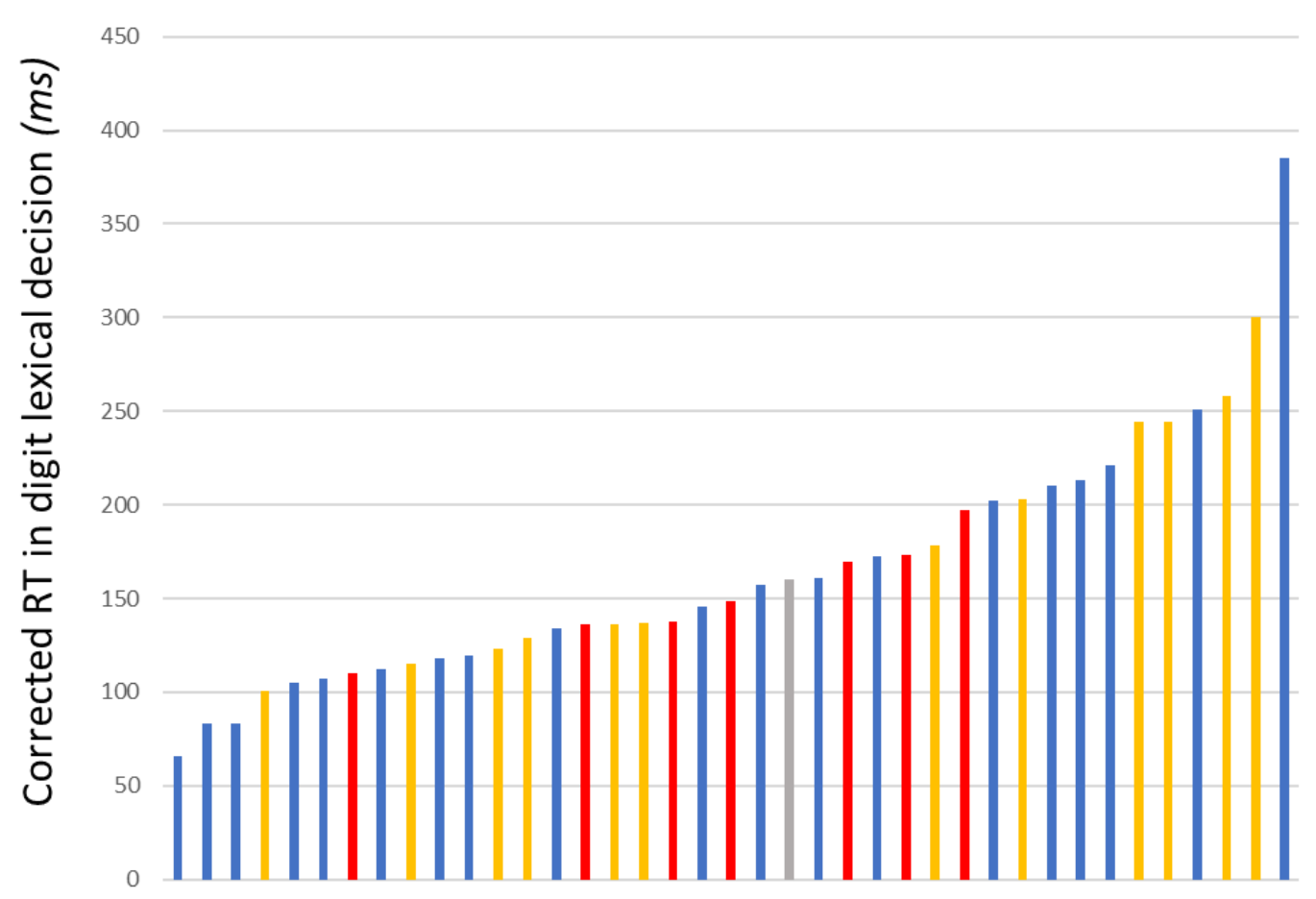

Participants

Figure 5. Difference in reaction time (RT) between the digit lexical decision task and the matched choice reaction time task, for all the participants, ranged from the lowest to the highest. In blue: control participants; in light grey: mean of the control participants; in red: the seven participants with mathematical learning disability (MLD) identified as out of the range of controls in the Arabic digit comparison task; in yellow: other participants with MLD.

The second ANOVA, with lexical decision Task (digits vs. letters) and Background (transparent vs. noisy) as within-subjects factors and Group (MLD vs. CG) as between-subjects factor, revealed a main effect of Background $\left(F(1,36)=310.06, p<.001, \eta^{2}=.896\right)$, with faster RTs in the transparent $(M=544 \mathrm{~ms}, S D=68 \mathrm{~ms})$ than in the noisy conditions $(M=616 \mathrm{~ms}, S D=75 \mathrm{~ms})$. There was no effect of the Task $(F(1,36)=.28, p=.603)$, but a significant interaction between Task and Background $(F(1,36)$ $\left.=4.21, p=.047, \eta_{\mathrm{p}}^{2}=.105\right)$. Post-hoc comparisons showed that transparent stimuli are answered faster than noisy ones in both lexical decision tasks (DIGIT-DECISION: $t(37)=-12.72, p<.001$; LETTERDECISION: $t(37)=17.16, \mathrm{p}<.001)$. However, the difference in RT between the two backgrounds was larger in the LETTER-DECISION $(M=78 \mathrm{~ms}, S D=33 \mathrm{~ms})$ than in the DIGIT-DECISION $(M=66 \mathrm{~ms}, S D=$ $33 \mathrm{~ms} ; t(37)=-2.54, p<.048)$. Finally, we found a main effect of Group $\left(F(1,36)=11.17, p<.001, \eta^{2} \mathrm{p}\right.$ $=.237)$, indicating that $\mathrm{CG}$ participants $(M=548 \mathrm{~ms}, S D=75 \mathrm{~ms})$ responded faster than MLD 
participants $(M=612 \mathrm{~ms}, S D=72 \mathrm{~ms})$, but this factor did not interact with Task and/or Background factors (i.e., all p-values > .122).

\section{Discussion}

The results show that, when they have to compare the magnitude of Arabic digits, adults with MLD take more time than typically developed people, and this increase in response latency cannot be explained by attention-, decision- or response-related processes that were carefully controlled in a matched choice reaction time task used as a baseline. A fine-grained analysis of individual data indicated that 7 out of the 19 participants with MLD exhibited a larger slowdown in the Arabic digit comparison task than what could be expected from their response latency in the matched choice reaction time task. The main implication is that the difficulties experienced by children with MLD maintain over development until adulthood (De Smedt \& Gilmore, 2011; Landerl \& Kölle, 2009; Rousselle \& Noël, 2007; Wilson et al., 2015). Hence, adults with MLD who have achieved high school graduation and are regularly confronted with symbolic numbers may still face a cognitive cost to perform numerical judgements on them. In particular, the results suggest that the poor arithmetic skills of adults with MLD may actually hide a primary deficit in Arabic digit processing, which is likely to affect a wide range of basic numerical activities in everyday life.

In contrast, our study showed that adults with MLD show an intact ability to recognize that a symbol is an Arabic digit - no matter its identity or numerical meaning. Their performance in an Arabic digit lexical decision task does not differ from the typically developed adults, after controlling for response speed differences using a matched choice reaction time task as a baseline. This finding holds even when contrasting digit and letter recognition, or trials of different levels of difficulty (noisy vs. transparent background). Hence, the second implication of our results is that adults with MLD experience difficulties in the processing of Arabic digits, despite efficient digit visual recognition. The main effect of visual noise on RTs proves that the lexical decision task is sensitive to the perceptual context and should reflect a core deficit in visual digit recognition if it was present. The digit lexical decision task also proved to be as difficult as the digit comparison task if one considers that participants 
took more time to discriminate digits from pseudo-digits than to compare the magnitude of two digits. This observation supports the external validity of our assessment of Arabic digit processing because a similar difference was observed between digit comparison and lexical decision in the study of Lafay and colleagues (2018). This RT difference could be due to the fact that the magnitude comparison task was more familiar to the participants. It could also be due to the fact that the magnitude comparison task required pressing the key on the side of the largest number (e.g., left or right side), whereas the lexical decision task involved arbitrary stimulus-response mapping (e.g., left if digit or right if pseudo-digit). The latter may also explain the RT difference observed between the two choice reaction time tasks that also differed in response mapping.

The present results thus contradict the hypothesis that the difficulties of adults with MLD in Arabic digit processing find their origin in a pre-semantic deficit affecting digit visual recognition. In their study, Lafay and colleagues (2018) reported that children with MLD were less accurate than their peers in a lexical decision task on Arabic digits. We see two possible explanations for these discrepant results. First, no difference in digit lexical decision was observed between children when scores in nonverbal reasoning and linguistic measures were used as a covariate in the statistical analysis. If the effect of MLD on lexical decision was mediated by reasoning skills in this previous study, then it is no surprise that adults with MLD show no lexical decision impairment in our study since the inclusion criteria required them to achieve standard performance on the verbal reasoning test "Similitudes" from the WAIS-IV (Wechsler, 2011). Although the mediating role of reasoning skills puts limits on what can be inferred from the comparison of the two studies, another explanation exists which considers that a difficulty to recognize Arabic digits may compromise the acquisition of efficient numerical skills in childhood, leaving them durably affected even though the visual difficulty resolves later during development. This hypothesis predicts poor digit lexical decision performance in children with MLD, but not necessarily in adults with MLD since their digit visual recognition deficit may get attenuated over time with regular exposure to Arabic digits. However, the impact on numerical acquisitions would remain visible across lifespan, creating a gap in symbolic number knowledge, as suggested by the dissociated effect of MLD on magnitude comparison and lexical decision in our adult study. Further 
developmental studies are needed to investigate the crosstalk between the visual recognition of Arabic digits and the formation of magnitude representations across development, and to test the hypothesis of mutual interference during a critical period of acquisition. Decisive evidence could also come from training studies that aim to improve digit visual recognition skills in children while measuring the effect on other numerical performance. In the absence of further evidence, our data are best explained by the view that MLD is due to a semantic deficit affecting the processing stages where a meaning is assigned to numerical symbols. The deficit could be the expression of a general impairment of the ANS (Wilson $\&$ Dehaene, 2007), or a selective impairment affecting the exact representation of symbolic numbers (Marinova et al., 2021; Noël \& Rousselle, 2011; Roggeman et al., 2007) or the access to the representation of numerical magnitude from Arabic digits (Rousselle \& Noël, 2007). Our data are compatible with all these hypotheses, as far as they do not associate the impaired access or representation of digit magnitude with a dysfunction at the allographic level where a symbol is recognized as one of the digits.

Finally, our results converge with a number of studies showing that participants with MLD suffer from a recurrent slowdown in cognitive tasks, adding up to their difficulties with symbolic number processing, as evidenced here by the higher RTs observed in the control choice reaction time tasks (see also Censabella \& Noël, 2005; Lafay et al., 2018; Rousselle \& Noël, 2007; Wang et al., 2018). Domaingeneral deficits of cognitive control, attention, working memory or processing speed have been reported in MLD (Fias et al., 2013; Rubinsten \& Henik, 2009; Wang et al., 2018). In our and other studies in individuals with MLD (Cheng et al., 2018), the slowdown observed in non-numerical tasks that simply require matching low-level perceptual attributes with binary motor responses is indeed compatible with domain-general deficits affecting background cognitive processing. However, their nature and relationship to dyscalculia remains to be fully understood.

In conclusion, the present study showed that adults with a history of mathematical learning disability continue to experience difficulties not just in arithmetic tasks but also in basic numerical tasks such as digit comparison. We further showed that these difficulties are not associated with any impairment in the visual recognition of Arabic digits, as indexed by lexical decision performance, 
placing an important constraint on existing accounts. The possibility remains that a weakness in visual digit recognition may hinder the acquisition of efficient symbolic numerical skills in the early stages of development, before eventually resolving later on. This possibility calls for further investigation, in children in particular. 


\section{Acknowledgements}

The authors would like to warmly thank all the participants for their time, attention and kindness.

\section{Declaration of interest}

None.

\section{Funding}

The author(s) disclosed receipt of the following financial support for the research, authorship, and/or publication of this article: MA is a research associate at the Fonds National de la Recherche Scientifique (FRS-FNRS, Belgium); and SL is a doctoral student funded by a grant from the Fédération Wallonie Bruxelles (FWB, Belgium) [grant number ARC21/26-112]. 


\section{References}

American Psychiatric Association. (2013). Diagnostic and statistical manual of mental disorders (5th ed.). Washington, DC: Author.

Booth, J. L., \& Siegler, R. S. (2008). Numerical Magnitude Representations Influence Arithmetic Learning. Child Development, 79(4), 1016-1031. https://doi.org/10.1111/j.1467$\underline{8624.2008 .01173 . x}$

Carey S. (2009). Where Our Number Concepts Come From. The journal of philosophy, 106(4), 220254. https://doi.org/10.5840/jphil2009106418

Carey, S. (2001). Cognitive Foundations of Arithmetic : Evolution and Ontogenisis. Mind and Language, 16(1), 37-55. https://doi.org/10.1111/1468-0017.00155

Censabella, S., \& Noël, M.-P. (2005). The Inhibition of Exogenous Distracting Information in Children with Learning Disabilities. Journal of Learning Disabilities, 38(5), 400-410. https://doi.org/10.1177/00222194050380050301

Cheng, D., Xiao, Q., Chen, Q., Cui, J., \& Zhou, X. (2018). Dyslexia and dyscalculia are characterized by common visual perception deficits. Developmental Neuropsychology, 43(6), 497-507. https://doi.org/10.1080/87565641.2018.1481068

De Smedt, B., \& Gilmore, C. K. (2011). Defective number module or impaired access? Numerical magnitude processing in first graders with mathematical difficulties. Journal of Experimental Child Psychology, 108(2), 278-292. https://doi.org/10.1016/j.jecp.2010.09.003

De Smedt, B., Noël, M.-P., Gilmore, C., \& Ansari, D. (2013). How do symbolic and non-symbolic numerical magnitude processing skills relate to individual differences in children's mathematical skills? A review of evidence from brain and behavior. Trends in Neuroscience and Education, 2(2), 48-55. https://doi.org/10.1016/j.tine.2013.06.001

De Visscher, A., \& Noël, M.-P. (2013). A case study of arithmetic facts dyscalculia caused by a hypersensitivity-to-interference in memory. Cortex, 49(1), 50-70. https://doi.org/10.1016/j.cortex.2012.01.003 
de Vos, T. (2010). TempoTest Automatiseren. Boom Test Uitgevers.

Dehaene, S. (1992). Varieties of numerical abilities. Cognition, 44(1), 1-42. https://doi.org/10.1016/0010-0277(92)90049-N

Dehaene, S., \& Cohen, L. (1995). Towards an anatomical and functional model of number processing. Dans B. Butterworth (Ed.), Mathematical cognition (Vol.1, pp. 83-120). Psychology Press.

Fias, W., Menon, V., \& Szucs, D. (2013). Multiple components of developmental dyscalculia. Trends in Neuroscience and Education, 2, 43-47. https://doi.org/10.1016/j.tine.2013.06.006

Geary, D. C. (2004). Mathematics and Learning Disabilities. Journal of Learning Disabilities, 37(1), 4-15. https://doi.org/10.1177/00222194040370010201

Geary, D. C., Hamson, C. O., \& Hoard, M. K. (2000). Numerical and arithmetical cognition: a longitudinal study of process and concept deficits in children with learning disability. $J$ Exp Child Psychol, 77(3), 236-263. https://doi.org/10.1006/jecp.2000.2561

Iuculano, T., Tang, J., Hall, C. W. B., \& Butterworth, B. (2008). Core information processing deficits in developmental dyscalculia and low numeracy. Developmental Science, 11(5), 669-680. https://doi.org/10.1111/j.1467-7687.2008.00716.x

Lafay, A., Macoir, J., \& St-Pierre, M.-C. (2018). Impairment of Arabic- and spoken-number processing in children with mathematical learning disability. Journal of Numerical Cognition, 3(3), 620-641. https://doi.org/10.5964/jnc.v3i3.123

Landerl, K., \& Kölle, C. (2009). Typical and atypical development of basic numerical skills in elementary school. Journal of Experimental Child Psychology, 103(4), 546-565. https://doi.org/10.1016/j.jecp.2008.12.006

Landerl, K., Bevan, A., \& Butterworth, B. (2004). Developmental dyscalculia and basic numerical capacities: a study of 8-9-year-old students. Cognition, 93(2), 99-125. https://doi.org/10.1016/j.cognition.2003.11.004 
Marinova, M., Sasanguie, D., \& Reynvoet, B. (2021). Numerals do not need numerosities: robust evidence for distinct numerical representations for symbolic and non-symbolic numbers. Psychological Research, 85(2), 764-776. https://doi.org/10.1007/s00426-019-01286-Z

Mazzocco, M. M. M., Feigenson, L., \& Halberda, J. (2011a). Preschoolers' Precision of the Approximate Number System Predicts Later School Mathematics Performance. PLoS ONE, 6(9). https://doi.org/10.1371/journal.pone.0023749

Mazzocco, M. M. M., Feigenson, L., \& Halberda, J. (2011b). Impaired Acuity of the Approximate Number System Underlies Mathematical Learning Disability (Dyscalculia) : Impaired Numerical Acuity Contributes to MLD. Child Development, 82(4), 1224-1237. https://doi.org/10.1111/j.1467-8624.2011.01608.x

McCloskey, M., \& Schubert, T. (2014). Shared versus separate processes for letter and digit identification. Cognitive Neuropsychology, 31(5-6), 437-460. https://doi.org/10.1080/02643294.2013.869202

McCloskey, M., Caramazza, A., \& Basili, A. (1985). Cognitive mechanisms in number processing and calculation : Evidence from dyscalculia. Brain and Cognition, 4(2), 171-196. https://doi.org/10.1016/0278-2626(85)90069-7

Noël, M.-P., \& Rousselle, L. (2011). Developmental Changes in the Profiles of Dyscalculia : An Explanation Based on a Double Exact-and-Approximate Number Representation Model. Frontiers in Human Neuroscience, 5. https://doi.org/10.3389/fnhum.2011.00165

Peirce, J. W. (2019). PsychoPy (Version 3.1.5) [Logiciel]. Pierce. https://github.com/psychopy/psychopy/releases?after=3.2.2

Piazza, M., Facoetti, A., Trussardi, A. N., Berteletti, I., Conte, S., Lucangeli, D., Dehaene, S., \& Zorzi, M. (2010). Developmental trajectory of number acuity reveals a severe impairment in developmental dyscalculia. Cognition, 116(1), 33-41. https://doi.org/10.1016/j.cognition.2010.03.012 
Piazza, M., Pica, P., Izard, V., Spelke, E. S., \& Dehaene, S. (2013). Education Enhances the Acuity of the Nonverbal Approximate Number System. Psychological Science, 24(6), 1037-1043. https://doi.org/10.1177/0956797612464057

Reynvoet, B., \& Sasanguie, D. (2016). The Symbol Grounding Problem Revisited: A Thorough Evaluation of the ANS Mapping Account and the Proposal of an Alternative Account Based on Symbol-Symbol Associations [Review]. Frontiers in Psychology, 7. https://doi.org/10.3389/fpsyg.2016.01581

Roggeman, C., Verguts, T., \& Fias, W. (2007). Priming reveals differential coding of symbolic and non-symbolic quantities. Cognition, 105(2), 380-394. https://doi.org/10.1016/j.cognition.2006.10.004

Rousselle, L., \& Noël, M.-P. (2007). Basic numerical skills in children with mathematics learning disabilities : A comparison of symbolic vs non-symbolic number magnitude processing. Cognition, 105(3), 361-395.

Rubinsten, O., \& Henik, A. (2005). Automatic Activation of Internal Magnitudes : A Study of Developmental Dyscalculia. Neuropsychology, 19(5), 641-648. https://doi.org/10.1037/0894$\underline{4105.19 .5 .641}$

Rubinsten, O., \& Henik, A. (2009). Developmental dyscalculia: heterogeneity might not mean different mechanisms. Trends Cogn Sci, 13(2), 92-99. https://doi.org/10.1016/j.tics.2008.11.002

Schubert, T., \& McCloskey, M. (2013). Prelexical representations and processes in reading: Evidence from acquired dyslexia. Cognitive Neuropsychology, 30(6), 360-395. https://doi.org/10.1080/02643294.2014.880677

Schwenk, C., Sasanguie, D., Kuhn, J. T., Kempe, S., Doebler, P., \& Holling, H. (2017). (Non)symbolic magnitude processing in children with mathematical difficulties: A meta-analysis. Research in developmental disabilities, 64, 152-167. https://doi.org/10.1016/j.ridd.2017.03.003 
Shalev, R. S., Auerbach, J., Manor, O., \& Gross-Tsur, V. (2000). Developmental dyscalculia: prevalence and prognosis. European child \& adolescent psychiatry, 9 Suppl 2, II58-II64. https://doi.org/10.1007/s007870070009

Shalev, R. S., Manor, O., Kerem, B., Ayali, M., Badichi, N., Friedlander, Y., \& Gross-Tsur, V. (2001). Developmental Dyscalculia Is a Familial Learning Disability. Urban Education, 34(1). https://doi.org/10.1177/00420859211017978

Sullivan, K. S. (1996). Remediation of arabic numeral processing in a case of developmental dyscalculia. Neuropsychol Rehabil, 6(1), 27-54. https://doi.org/10.1080/713755495

Temple, C. M. (1989). Digit dyslexia: A Category-specific disorder in development dyscalculia. Cognitive Neuropsychology, 6(1), 93-116. https://doi.org/10.1080/02643298908253287

Wang, X., Georgiou, G. K., Li, Q., \& Tavouktsoglou, A. (2018). Do Chinese Children With Math Difficulties Have a Deficit in Executive Functioning? Frontiers in Psychology, 9, 906. https://doi.org/10.3389/fpsyg.2018.00906

Wechsler, D. (2011). WAIS-IV - Échelle d'intelligence de Wechsler pour adultes (5 éd.). ECPA.

Wilson, A. J., \& Dehaene, S. (2007). Number sense and developmental dyscalculia. Dans D. Coch, G. Dawson, \& K. W. Fischer (Eds.), Human behavior, learning, and the developing brain : Atypical development (pp. 212-238). The Guilford Press.

Wilson, A. J., Andrewes, S. G., Struthers, H., Rowe, V. M., Bogdanovic, R., \& Waldie, K. E. (2015). Dyscalculia and dyslexia in adults : Cognitive bases of comorbidity. Learning and Individual Differences, 37, 118-132. https://doi.org/10.1016/j.lindif.2014.11.017

Zhou, X., Wei, W., Zhang, Y., Cui, J., \& Chen, C. (2015). Visual perception can account for the close relation between numerosity processing and computational fluency. Front Psychol, 6, 1364. https://doi.org/10.3389/fpsyg.2015.01364 